\title{
Freelancing System
}

\author{
Chamathka Dissanayake, Dehami Rathnayake, Nadeesha Dilhani, Udeni Kumarasiri, Nandani Kumari \\ K.A. Dilini T Kulawansa
}

\begin{abstract}
Freelancing is doing a job under contract rather than full employment. Usually, people who freelance are selfemployed and for whom they will work and they decide for how long. Freelancing offers the lot of freedom and flexibility which regular jobs cannot. The freelancer can choose the type of the work, time of the day can work and how long they work, a location they work and volume of work take on. The freelancer can control work relationship and control workload. Platform freelancing has many websites and anyone can register as freelancers or job posters. Sri Lankan service providers register as freelancers through these websites. there is no local freelancing platform for Sri Lankan freelancers yet. They register through foreign freelancing platforms. A major issue of these systems is Sri Lankan freelancers cannot get gross profit for their services. Foreign freelancing sites charge a higher percentage of their revenue as service charges. The system is developed to overcome these drawbacks. The system is designed only for Sri Lankan freelancers to hold their revenue within the country. there is a discussion forum to build up friendly environment between freelancers and clients. all they can work without any language issue. For these solutions, the system is designed according to four submodules such as user validation module, file sharing module and manage profile. Users of the system are Job poster, Freelancer (Jobseeker). Inputs of the system are Details of the Job poster, Freelancer, Job, gig. Outputs of the system are Overall rating, feedback, and notification. Processes of the system are collected data, analyze data and job posting, creating gigs, discussion and complete the job. Technologies used for the system are Angular CLI, JavaScript, HTML, CSS, PHP, MySQL, Node Js. In the analysis and design section, the system is designed using the EER diagram and UML diagrams. Level of user satisfaction is evaluated by using a questionnaire. The questionnaire has consisted of 8 criteria. It is distributed among 40 selected users and collects their feedback.
\end{abstract}

Keywords_- Freelancing, Freelancer

\section{Introduction}

Simply, freelancing is doing a job under contract rather than full-time employment. Nowadays freelancing is more popular among people. [10] Especially among creative workers. Therefore, we decided to develop a freelancing system. The main intention of our project is providing the platform which is only Sri Lankan can register as service providers. Because when they use foreign freelancing systems, they bereave a fragment of their payment or wages. So, through our system, we can keep domestic revenue in our country. Freelancing systems open the huge range of career opportunities to workers rather than attached to the single company and our system also gives all these facilities to freelancers but the specification is all they are Sri Lankans.

When we pay our attention to the most basic question "Why we need freelance?" regarding to our system we can identify many reasons for this question. Some of them are the Economy of Sri Lanka needs more freelancers., Business prefers to hire freelancers.

the other thing is when people tend to use freelancing, they can identify significant variation and flexibilities. Basically, those significant variation flexibilities can divide into four main categories. Those are duration, type of work payment, source work. According to the duration, some contracts are short a few hours, some are month to month and some are year to year. According to a type of work some are low skill job, some are projects and team-based jobs and some require substantial expertise and certification. People now like to do freelancing they would be able to work under this any types. The payment also can various types. Some are small hourly wages, some are fixed price projects and some mimic a full-time salary. When we considering the source of work of freelancing it can be done over freelance broker sites or direct sourced. As well as some are cooperating and nearly indistinguishable from fulltime works. [10]

In order to our system build up a platform to freelancers to do their freelancing over freelancing broker site with all above significant variation flexibilities. So, Sri Lankan freelancers can be a standalone worker and make own decisions rather than cope with any other persons.

Our system is open to everyone who registers as customers and they have a facility to select freelancer by using rating system or bedding system as they wish.

\section{Related Work}

Our software system is the freelancing system. There are so many similar projects like ours in Sri Lanka as well as in other countries. Because nowadays freelancing is more popular among people [12]. Before we develop our own system, we decided to find the details of other similar systems. We were identified software systems that have already been developed as freelancing systems. We deeply 
go through the features, facilities, user comments of other freelancing systems that have already implemented. Our expectation is to gather information and after gathering that information, we can compare our system with those systems and can recognize drawbacks in our system. We can study about their advantages and disadvantages. We can remove those drawbacks from our system and can add new values to the system. Then we would able to develop a system which overcomes these identified drawbacks. At the end, we will be able to present product which can ensure all the functions that should be in freelancing system. To develop a more accurate system we study five similar systems namely Crew, Guru, Elance, 99design, Fiver. All these systems have the same goal of developing a platform to connect freelancers and job posters with different other features.

However, Sri Lankan freelancers do not have their own platform for freelancing. Our main intention has developed a system with the all identified function of other similar systems and which is only Sri Lankan register as freelancers.

\section{Technologies Adopted}

The purpose of our software project is to create a freelancing system for the only register can Sri Lankan freelancers. In our project, there is one database which stores information about the users. We used MYSQL to create the database. In this database, we stored the user's name, id, jobs, job id, gigs and gig id etc. Through this system, a user can register as a freelancer or job poster. After the registration job poster can create the profile, search freelancers and add the job. As well as the freelancer also can create the profile, search jobs, and job posters and post gig. In addition to that, both freelancer and job poster can chat using the discussion forum. The web interface developed in our system by using Angular CLI and Bootstrap

\section{Modular Approach To Freelancing System}

There are many operations over a freelancing system. The system contains with many modules in an application. These modules complete the basic tasks of our system. These all modules are slightly different and each module dedicates for specific functionality. The interconnection between those modules course to obtain all the tasks complete successfully. All those modules consist with a number of submodules and it should be properly bound to get the correct output from the system [28]. Otherwise, the system can be failed. As well as some modules have superior attributes and behaviors. Those modules reuse in several times where they can apply. Since this reason, the system can avoid the complexity [30].

Our proposed system consists of 6 modules, namely, login module, create a profile module, create a gig module, discussion forum module and add job module. There are two types of users such as freelancer and the job poster. Before login to the system they have to register as freelancer or job poster. Registration is verified through the email. After registration, they can $\log$ in to the system and create a profile. After login as freelancer he or she can create gigs. There are different packages in the gig and job poster can select one of the gigs from it. And also, the freelancer can update the gigs. Job poster can add the job. Discussion forum between freelancer and client. After freelancer accept the job both freelancer and client can discuss price, the time period of the job and everything they want to know each other.

\section{v. Analysis And Design}

With the development of technologies and increment of advancement of problem addressing people tend to use diagrams to simplify the problem. Especially IT related problems do a major role in this case. Using UML diagrams, we can clearly identify the logic behind the problem. Use case diagram illustrates functionalities provided by the system and functional requirements of a system by including actors and relationship among use cases [17].

The class diagram shows different entities, static structure and implementation of classes. The sequence diagram shows the detailed flow of use cases in detailed. Activity diagram interprets a procedural flow of control between two or more classes while doing and acting. Context diagram classifies the boundary between the system and its environment how it interacts with entities. ER diagram illustrates relationships of entities stored in the database, the cardinality of them and how data is related to each other. The actual functionality of the system is delivered in the analysis. Architecture of the system can be seen from "Figure 1".

Registration- In our system registration is happening in two ways. They are freelancers who willing to finish jobs and Job posters who post jobs. Freelancers can post gigs and Job posters can post jobs through the system.

Gig- Gigs are posted by Freelancers. One freelancer can post their gigs with full description of what they can do as a freelancer. A include work, charge, duration and its duration. 
Job- Jobs are posted by Job posters. After Job posters posting jobs Freelancers can accept jobs. For posting a job, Job posters should include a description with requirements.

Discussion Forum- The system includes a discussion forum for more discussion between Freelancers and Job posters. Through this discussion forum, they can continue the work.

Data Base- Data Base include all information about Freelancers, Job posters, Gigs, Jobs, Discussion, rating

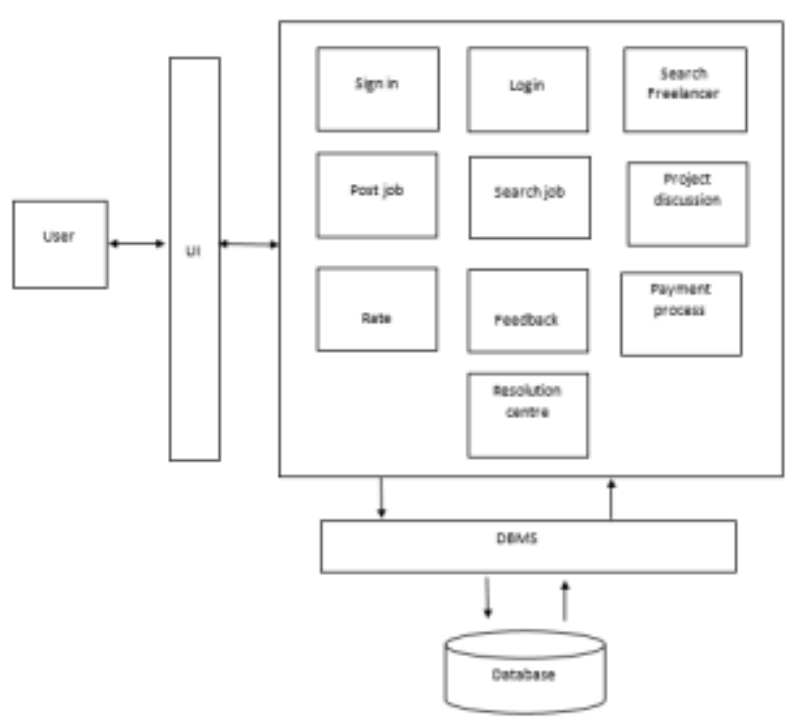

Figure 1. Top Level Architecture of the proposed system

\section{vi. Implementation of Our Proposed System}

First of all, we gathered requirements to start our project by visiting our company. Then we identified the major features of a freelancing system. Then we designed our system as per the requirements. Initially, we designed the ER diagram. Then we designed a use case diagram, Activity diagram, sequence diagram, class diagram. We used the knowledge of object-oriented analysis and design, software engineering methods to create this diagram.

System has two types of users. They are freelancer and job poster We have implemented web interfaces for the freelancer and the job poster using Anguler4. Sri Lankan can register as a freelancer in the system by creating profile and Foreigners can't register as the freelancer. IP address verifies by the system to identify Sri Lankan users. Anyone can register as a job poster. Users who wish to register to the system can enter to the system through the web interface and enter the details and submit it. There is a verification system. Verification code sent to user email address and after verify user can log in to the system. Registered users can view freelancers, jobs, job posters through a web interface. A registered user can easily sign up to the system using the username and the password. When a user enters the details, there is a verification system to identify the user.

When user login as a freelancer they can search for the available jobs and job poster. After selecting the job or job poster, the freelancer can easily check the details of the job and apply for the job. The registered freelancer can post the gig and update their profile any time by login to the system. job poster can view available freelancers and gigs posted by the freelancer. They can post the job and update their profile. The discussion forum was implemented to do project discussion between freelancer and job poster.

\section{Evaluation}

Our system is mainly privileged by job posters and freelancers. The performance of the system was tested using 40 users, in eight different evaluation aspects and responses were taken as a five-stage rating. The questionnaire has been evaluated the customer comments on different aspects such as user-friendliness of the application, Concept of the application, responding speed of the system, Accuracy level of the system, the business model for the system, usefulness of the application for the developing countries and use of modern technologies.

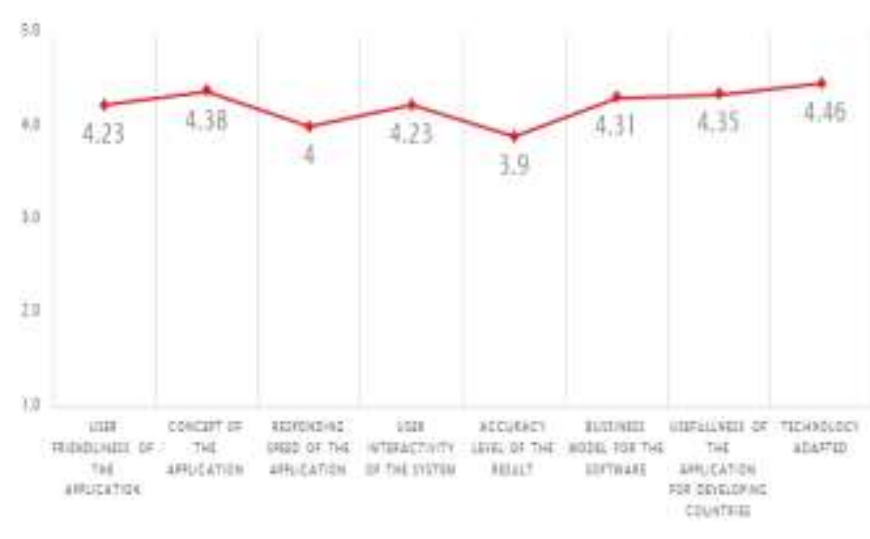

Figure 2. Graphical summary of user feedback

The statistics revealed that the system has its maximum rating for use of modern technologies which is 4.46. Almost all the factors we checked about the system had got ratings above 4.00 except one factor that is the accuracy level of the result.

It can be seen from Figure 2 that the average rating for the first evaluation aspect: User friendliness of the application is about 4.23, which lies between 4 and 5, where "4" stands for "Good" and "5" stands for "Very Good". Likewise, the average rating for the second evaluation aspect: Concept of the application is about 4.38 and it also lies between 4 and 5 . Third evaluation aspect is the responding speed of the application is about 4 average rating where " 4 " stands for "Good". User interactivity of the system is about 4.23 average rating, fifth evaluation aspect: Accuracy level of the 
system, which is about 3.90 average rate, lies between 3 and 4. That aspect has the lowest average rating among other ratings. Average rating for the business model for the software is about 4.31 and aspect number seven: Usefulness of the application for the developing counties is about 4.35. 4.46 rating is has got for use of modern technologies. This application, which was developed as a proof of concept has got 4.23 out of 5 as the average rating from all aspects. "TABLE 1" showed evaluation aspects of the system.

TABLE 1. Evaluation Aspects

\begin{tabular}{|c|c|c|c|c|c|c|c|}
\hline & Evaluafion aspect & $\begin{array}{l}\text { Very } \\
\operatorname{gocd}\end{array}$ & Good & Satisfactory & poor & $\begin{array}{l}\text { Very } \\
\text { poor }\end{array}$ & $\begin{array}{l}\text { Arerage } \\
\text { rating }\end{array}$ \\
\hline 1 & User friendliness of the appictifion & 16 & 17 & 7 & 0 & 0 & 4.23 \\
\hline 2 & Concept of the application & 16 & 23 & 1 & 0 & 0 & 4.38 \\
\hline & Responding speed of the application & 8 & 23 & 8 & 0 & 0 & 4 \\
\hline 4 & User interactivity of the system & 15 & 19 & 6 & 0 & 0 & 4.23 \\
\hline 5 & Accuracy level of the result & 8 & 20 & 12 & 0 & 0 & 3.9 \\
\hline 6 & Business model for the sottuare & 15 & 21 & 3 & 0 & 0 & 4.31 \\
\hline & $\begin{array}{l}\text { Usefiuness of the application for } \\
\text { developing comntries }\end{array}$ & 22 & 12 & 4 & 2 & 0 & 4.35 \\
\hline 8 & Use of modem technologies & 18 & 21 & 0 & 0 & 0 & 4.46 \\
\hline
\end{tabular}

\section{Limitations}

There are many freelancing systems available in the world. And they have many users that actively participate with this system to give their service or take some service. But actually, Sri Lankan freelancers haven't owned website to give their service and earn the gross profit. So, we decided to develop a freelancing platform for Sri Lankan freelancer. However, when considering our system boundaries. There are few limitations have been identified that we can't be achieved as follows. Inability to access foreign freelancers and System is based on the English language.

\section{Further Work}

Our proposed freelancing system can be further improved by adding the mobile app. By analyzing user feedback, we realized that suggestions which were given by users can be further improved such as develop an SMS notification system.

\section{$x$. Conclusion}

Internet becoming an obligatory factor while the complexity of the lifestyles of human beings is increasing day by day since the internet plays a major role in facilitating people to make their workload done efficiently. Freelancing is one of the technologies already available in the world. As well as there are many freelancing platforms. Such as Upwork, guru, fiver etc. using these websites anyone can register as buyer or seller. According to Sir Lankan freelancers also can register on these websites. But can't get gross profit for their services. Therefore, we decided to develop a system for Sri Lankans. Presentation layer has been implemented by HTML, Bootstrap, Anguler4. We have used MySQL to develop the Server Side. The users of our system are freelancer and job poster. The inputs of our system are such as add and remove freelancer and job poster with details, modify details of freelancer and job poster, add remove and modify jobs and gigs. The outputs of our system are namely Overall Rating, feedback. There are several processes in our system which are Collect data, analyze data, Job posting, Creating Gigs, Discussion, Complete the job. We have clearly demonstrated how our system works using UML diagrams. When implement we use a modular approach.

\section{Acknowledgment}

We wish to thank our supervisor for guiding us, giving stimulating suggestions and making encouragement among us and also helping us to make this research a success. we would like to deeply thank to all respondent who responded to our questionnaire and many individuals; friends and colleagues who supported us to success this research. Finally, everybody who helped us in various ways are gratefully acknowledged.

\section{References}

[1] Andrew Carrie,(July 16, 2015) Freelancer Management Systems: The Origin and Destiny of any Species

[2] Addrew Burke,Handbook of research on freelancing \& selfemployment

[3] Deve cohen,(August 2011),How to become a successful Freelance

[4] Ed Gandia,(August 2012), Data and Analysis of Freelancer Demographics, Earnings, Habits and Attitudes

[5] Phillip S.leventhel ,(2009),Freelancer or Employee. Which is better?,

[6] Reeves A, Hamilton S. EMWA ,Freelance Business Survey 2007

[7] https://www.slideshare.net/cameronrambert1/be-your-own-boss-anintroduction-to-freelancing

[8] https://en.wikipedia.org/wiki/Freelancer

[9] https://www.freelancersunion.org/

[10] https://www.udemy.com/introduction-to-freelancing/learn/v4/

[11] http://www.quora.com

[12] https://en.wikipedia.org/wiki/Fiverr

[13] https://www.sitejabber.com/reviews/www.fiverr.com

[14] https://99designs.com 
Proc. of the Eighth Intl. Conf. on Advances in Computing, Electronics and Communication - ACEC 2019

Copyright $($ Institute of Research Engineers and Doctors. All rights reserved.

ISBN: 978-1-63248-165-8 DOI : 10.15224/978-1-63248-165-8-11

[15] https://it.wikipedia.org/wiki/99designs

[16] www.guru.com

[17] https://crew.co

[18] https://en.wikipedia.org/wiki/Elance

[19] https://www.fiverr.com

[20] https://www.nateshivar.com/486/99-designs-review-with-pros-consof-crowdsourced-logo- design

[21] https://en.wikipedia.org/wiki/Crew

[22] https://en.wikipedia.org/wiki/Guru

[23] https://www.elance.com

[24]https://www.w3schools.com/js/default.asp
[25]

[26]https://www.sitepoint.com/whats-new-in -angularjs-2/

[27]https://en.wikipedia.org/wiki/AngularJS

[28]https://www.youtube.com/watch?v=9CcpAMipA48

[29] http://www.kodingmadesimple.com

[30] http://searchsqlserver.techtarget.com/definition/SQL-Server-2014

[31] http://www.fiverrscript.com/local-bank-payments-module

[32] http://www.fiverrscript.com/custom-order-module

[33] https://en.wikipedia.org/wiki/Modular design 